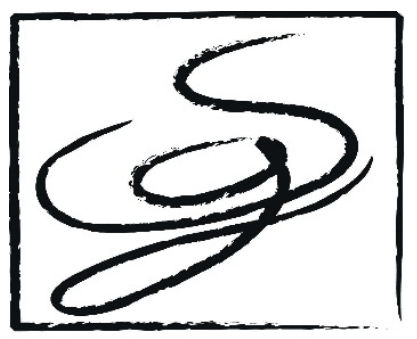

SEMINARIO DE INUESTIGACIÓN DE GÉNERO Y ESTUDIOS GULTURALES

\section{Enfance et didactique des identités de genre dans Plus sage que le roi de Béatrice Laure Mebou}

\author{
Childhood and didactic of gender identities in \\ Béatrice Laure Mebou's Plus sage que le roi \\ Pierre Suzanne Eyenga Onana \\ Université de Yaoundé I \\ eyenga_pierre@yahoo.fr
}

Fecha de recepción: 06/07/2021 Fecha de evaluación: 09/10/2021

Fecha de aceptación: 15/11/2021

\begin{abstract}
Written storytelling offers itself in Africa as an essential vector in the holistic education of the masses in ethics. The child sometimes plays the role of the wise teacher, working to renew gender identities in the face of the patriarchy that prevails. What strategies does he or she use to postulate new gender relations? Pierre Barbéris' sociocriticism guides this reflection, which is organized in two parts. First, we explore the explicit by questioning the didactics underlying the child's approach. Then, thanks to the implicit, we examine the aesthetics that structure the production of meaning and found the discourse on the world of Béatrice Laure Mebou.
\end{abstract}

Key words: written storytelling, child, sociocritic, gender, aesthetics, ethics

\title{
Resumen
}

En África, el cuento escrito es un vector esencial en la educación integral de las masas en materia de ética. El niño a veces desempeña el papel de sabio educador, trabajando para renovar las identidades de género frente al patriarcado imperante. ¿Qué estrategias utiliza para postular nuevas relaciones sociales de género? La sociocrítica de Pierre Barbéris guía la presente reflexión, que se organiza en dos partes. En primer lugar, se explora lo explícito cuestionando la didáctica que subyace al enfoque del niño. A continuación, gracias a lo implícito, examinamos la estética que estructura la producción de sentido y fundamos el discurso sobre el mundo de Beatrice Laure Mebou.

Palabras clave: cuento escrito, niño, sociocrítica, género, estética, ética 


\section{Résumé}

Le conte écrit s'offre en Afrique comme un vecteur essentiel dans l'éducation holistique des masses à l'éthique. L'enfant y joue parfois le rôle du didacticien avisé, travaillant au renouvellement des identités de genre face au patriarcat ambiant. Quelles stratégies convoque-t-il pour postuler des rapports sociaux de genres neufs ? La sociocritique de Pierre Barbéris oriente la présente réflexion organisée en deux parties. D'abord, on explore l'explicite en questionnant la didactique qui sous-tend la démarche de l'enfant. Ensuite, grâce à l'implicite, on scrute l'esthétique qui structure la production du sens et fonde le discours sur le monde de Béatrice Laure Mebou.

Mots clés : conte écrit, enfant, sociocritique, genre, esthétique, éthique

\section{Introduction}

S'exprimant sur la transmission populaire au Cameroun, notamment sur le théâtre et ses différents supports, Alain Cyr Pangop affirme que « I'utilisation de supports et de moyens d'expression accessibles au grand nombre a su créer des langages et des univers propres » (2005: 74). L'acte de lecture participe desdites formes de langages parce que le message véhiculé par l'artiste transite de la parole ancestrale à l'écriture. Lire, de ce point de vue, revient à poser un geste d'envergure démocratique visant à s'affranchir voire se libérer des carcans de certaines pesanteurs. Le conte écrit sur l'enfant s'inscrit dans cette dynamique d'affranchissement des hommes prisonniers de leurs turpitudes à travers le discours qu'il déploie sur les identités de genre.

Comme le soulignent Pabe Mongo et al. dans Enfances, « l'enfance est une période enrichissante pour l'écrivain » (2005: $4^{\text {ème }}$ de couverture). Les contributeurs à cet ouvrage scrutent l'imaginaire infantile, allant ainsi " à la rencontre de leurs rêves, de leurs regrets, et parfois de leur tristesse la plus profonde. Mais, il y a aussi la joie, les jeux, la mère, les traditions, l'Histoire de l'Afrique qui défile sous [leurs] yeux » (Pabe Mongo, 2005: $4^{\text {ème }}$ de couverture). La littérature produite sur l'enfant interpelle autant le politique qu'elle s'intéresse à la politique, définie comme gestion des affaires de la cité. Car,

La lutte pour la vie - plutôt pour la survie - constitue la préoccupation absolue des peuples du TiersMonde actuellement, à telle enseigne qu'on est porté à croire que tout enfant qui naît dans ce monde apporte une note supplémentaire à la "symphonie" des cris de misère, de faim, d'oppression, de guerre, etc. (Bebey, 1989: $4^{\text {ème }}$ de couverture). 
L'importance de la littérature pour jeunes naît ainsi de l'urgence d'opérationnaliser l'idée que c'est l'éducation qui sauvera l'Afrique et la sortira des eaux troubles de l'enlisement économique et du sousdéveloppement. D'où la question rhétorique formulée à l'adresse de tout un continent par l'administrateur général de l'Agence Intergouvernementale de la Francophonie dans le sens de suggérer une démarche alternative aux formules qui jusque-là se sont révélées des leurres : « Et si maintenant on essayait l'éducation? N'est-il pas temps en effet de replacer l'alphabétisation et l'éducation de base comme $\mathrm{LA}^{1}$ priorité politique ? ". Certes, la littérature de jeunesse n'est pas encore parvenue à dévoiler tout le capital sémantique qui articule l'inventivité de ses auteurs dans le paysage de la création littéraire afférant à la culture africaine. On comprend pourquoi la thématique sur l'enfance demeure d'une actualité vivace dans le paysage littéraire. Dans L'Enfant de sable de Tahar Ben Jelloun, tout comme dans L'Enfant de la révolte muette de Camille Nkoa Atenga, l'enjeu de voir naître un enfant de sexe masculin au sein du foyer conjugal se trouve au cœur même de l'intrigue. De même, dans Une vie hypothéquée, c'est le désir de donner la vie à une fille qui sortira sa famille de la misère après une alliance prénatale conclue à son insu qui constitue l'essentiel de la trame du récit de Tcha Koura Sadamba. L'essai n'est pas en reste, qui ne mette l'enfant au centre des préoccupations heuristiques. Tel est le cas pour Le racisme expliqué à ma fille, où Tahar Ben Jelloun explique à l'enfant les tenants et les aboutissants du racisme comme gage d'hypothèque de la paix sociale.

La particularité de toutes ces productions réside dans la diversité des supports qui traitent de la question de l'enfant : il s'agit principalement du roman et de l'essai. En choisissant le conte écrit comme sous-genre de la littérature de jeunesse dans la perspective d'explorer la problématique de l'enfant-didacticien, B. Laure Mebou ne montre-t-elle pas que cette variante littéraire permet de sortir du giron qui lui sert de cadre d'expression traditionnel depuis l'époque des veillées autour du feu de bois dans les villages africains? En d'autres termes, comment le conte écrit investit-il d'autres angles d'attaque afférents au discours de l'enfant sur la question des inégalités de genre?

La sociocritique de Pierre Barbéris, ainsi que l'approche genre orientent l'analyse. Dans son mode opératoire, la sociocritique prône l'éclectisme et débouche sur une approche interdisciplinaire du texte scruté. Elle se construit à partir "d'une recherche et d'un effort tâtonnant et découvreur qui invente un nouveau langage, fait apparaître de nouveaux problèmes et pose de nouvelles questions" (Barbéris, 1990: 124). Autrement dit, « elle est attentive à tout ce qui émerge de nouveau, et qu'elle contribue à faire émerger, dans l'Histoire " (1990: 124-125). Deux axes opératoires en définissent l'épistémologie: la lecture de l'explicite et la lecture de l'implicite. S'agissant de l'approche genre, elle se fonde sur l'éloge du neutre qu'Eleni Varikas promeut dans son article «ni l'un ni l'autre. Eloge du neutre » (in Revue des Deux Mondes). L'approche genre

\footnotetext{
${ }^{1}$ C'est l'auteur qui souligne
} 
trouve son terreau fécond dans le constructionnisme. Les théoriciens de ce courant soutiennent qu' « il n'existe pas d'essence féminine ou masculine, mais seulement un sexe biologique qui n'influe pas ou très peu sur la personnalité ». (Nnomo, 2013: 23). L'approche genre construit ainsi son sémantisme autour d'un impératif: "sortir du "carcan catégoriel" naturel/culturel, voir au-delà de la loi phallique qui fait du point de vue masculin, le point de vue objectif et savant et du point de vue féminin, un point de vue spécifique, signe de l'inférieur, du négatif, ou du mythe » (Calle-Gruber, 2003: 182).

L'analyse en soi comporte deux parties. La première, l'explicite, interroge les diverses modalités didactiques significatives auxquelles se trouve confronté l'enfant. La dernière, l'implicite, construit le sens en s'appuyant sur deux axes. D'abord, l'esthétique qui sous-tend la production du sens dans le texte mebousien ; ensuite, le discours sur le monde postulé par la nouvelliste.

\section{L'explicite ou l'Histoire au cœur d'une didactique différenciée}

Lire l'explicite revient à « recharger le texte de ce qui y est déjà, ['Histoire] mais qui a été marginalisé ou évacué. II ne s'agit pas ici d'une symbolique obscure, mais de références claires à restituer, et qui peuvent être disséminées » (Barbéris, 1990: 139). Lesdites références évoquent un fait marquant de l'Histoire tel qu'inscrit dans le récit : la réification du genre masculin. Ces références permettent de positionner le roman, tout comme la nouvelle ici considérée, comme micro-récit, c'est-à-dire " une sorte d'énoncé encyclopédique, mobilisant une description à la fois éclatée et cohérente de l'univers social, et disant l'Histoire ; soit effectivement une mimésis » (Mitterand, 1980: 8).

Dans le texte de Mebou, l'Histoire se dévoile à travers une esthétique du rejet de l'autre au prétexte que ce dernier représente une réelle menace contre le pouvoir établi ou la place du Roi. Pour disséquer l'inscription de l'Histoire dans la nouvelle mebousienne, on convoque le concept d'implicature conversationnelle qui relève de la pragmatique énonciative. Elle « implique le fait d'avancer un contenu $X$ dans l'intention consciente ou inconsciente de signifier une intention $Y^{2}$ ». Telle qu'elle se déploie dans le corpus, l'implicitation se révèle à travers le sous-entendu. On accède au sens par inférence. De nature contextuelle, et donc instable, le sousentendu permet d'établir qu'un énoncé actualisé dans des contextes différents suggère des sens différents.

L'un des sous-entendus est qu'en renouvelant son lieu d'opérationnalisation, le conte écrit articule une didactique inversée, voire renversée, puisque celle-ci n'est plus l'apanage de l'adulte savant professant ou pourvoyant des savoirs aux jeunes, mais bien celle de l'enfant. Au regard de sa posture de formateur, ce dernier inscrit les adultes, ses parents en premier, à l'école de la vie. Les actes qu'il pose sont empreints d'une expressivité exemplifiée qui vaut mieux que toute forme de

${ }^{2}$ In http://www.analyse-du-discours.com/l-implicitation consulté le 25 mai 2021. 
pédagogie traditionnelle. Deux déclinaisons teintées d'histoire articulent la nouvelle pédagogie qu'imprime le personnage éponyme dans Plus sage que le roi: l'onomastique et les réactions diverses aux actions du roi.

1.1. L'onomastique, un informant historico-didactique

Pour Milagros Ezquerro, «le personnage apparaît d'abord sous un nom » (1983: 121). Ce nom, en Afrique, est porteur de sens. Celui que porte le personnage central du conte Plus sage que le roi est révélateur de sa geste lorsqu'on appréhende cet indice onomastique au prisme de la didactique. L'enfant démontre à l'adulte, -un roi supposé plus avisé que tous-, que ses idées noires ne peuvent prospérer, surtout lorsqu'il s'entoure de conseillers mal intentionnés. Le nom devient un indicateur stratégique dans la dynamique des rapports sociaux de genre, tant il accompagne celui qui le porte et lui permet d'opérer un distinguo entre l'admissible ou le faisable et l'inconcevable. Ce nom en soi, se révèle être un motif de transmission des vertus réglementant les liens sociaux. L'une d'elle est le courage, comme cela se voit dans certaines productions romanesques.

Dans Une vie hypothéquée, Ya, la jeune fille vendue, fait preuve de courage en refusant d'épouser Béhira, le vieillard à qui l'ont mariée ses parents indigents, alors qu'elle n'était qu'un fœtus. De même, dans Le Fils d'Agatha Moudio, lorsqu'Agatha se trouve reniée par Moudio, son père, du fait d'être née fille au moment où ce dernier attendait un garçon à la quatrième grossesse de sa mère, elle choisira unilatéralement d'aller en mariage sans en référer à son géniteur pour des questions de dot. Elle défiera tout un village en épousant l'homme qu'elle aime. Cette option pour le courage est l'apanage des enfants qui souffrent parce qu'exposés à la violence sexiste autant qu'à la méchanceté des adultes dans un univers déjanté. Lorsque le roi lui demande son nom, l'enfant lui répond sans transiger : « je m'appelle Plus sage que le roi ! » (Mebou, 2009: 9). L'enfant enseigne aux adultes comment assumer leur nom et bien le porter, ce d'autant que pour Philippe Hamon, le nom est non seulement indispensable pour le personnage, mais il est surtout porteur d'une signification. La peur de se voir molesté par le roi aurait pu empêcher l'enfant de dévoiler son nom au chef du village. Son courage, à la limite de la témérité, le conduit pourtant à accepter les conséquences qui entourent son apparente imposture. La sanction du roi oblige l'enfant à faire montre d'une autre vertu : la bravoure.

Elle consiste pour l'enfant à assumer sans aucune gêne les sanctions que lui inflige le roi. La dureté desdites sanctions n'ont d'égale que l'insolence affirmée de l'enfant. Elle conduit le monarque à le faire prisonnier : " je ne te ferai pas mourir, mais tu te tueras toi-même. Rentre chez toi et dis à tes parents que désormais tu seras mon prisonnier » (2009: 9 ). Cette déclaration impose une triple modalité dans l'implémentation de la nouvelle pédagogie que déploie l'enfant face aux adultes. D'abord l'enfant, à l'instar de tous les administrés, a des droits que tout homme, fût-il roi, conviendrait de respecter. Ensuite, à moins qu'il ne soit un dictateur, le roi ne saurait se décrire comme un tueur de sang-froid promettant la mort à un enfant qui, pourtant, incarne l'avenir du village. Enfin, on ne saurait arbitrairement promettre la prison à un individu sans l'avoir au préalable 
jugé. De nombreuses promesses de mort rythment et ponctuent pourtant le discours du roi. Elles se voient à travers l'usage du champ lexical de la mort : « mourir »; « tueras ». Les divers plans que tisse le monarque pour faire trépasser l'enfant induisent un autre module de la pédagogie infantile : le rejet de l'autre. II consiste en l'activation des leviers par le genre dit dominant.

\subsection{L'Histoire et la question du genre}

Chez Mebou, le sous-entendu articule le sens des énoncés. II oblige à affirmer que c'est finalement la vision essentialiste qui domine les rapports sociaux de genre. Cette vision se manifeste notamment « une fois qu'on a décidé qu'une activité a un sexe, la participation de l'autre devient difficile et compromettante » (Mead, 1988: 428). Tel est le cas pour l'exercice de la fonction de roi au sein du pays « très lointain » qui constitue la toile spatiotemporelle dans la trame mebousienne. Le narrateur omniscient précise à cet égard que « les petits garçons n'avaient pas le droit de vivre " (Mebou, 2009: 7). Le sous-entendu qui traverse cette occurrence est que toute naissance masculine est ipso facto malvenue. Le Roi n'entend pas voir émerger une éventuelle concurrence qui conduirait à la perte de son trône. Le droit à la vie indiscriminée dont jouit à sa naissance tout enfant se trouve donc floué par ce monarque préoccupé de préserver les intérêts égocentriques qui l'animent. Le diktat qui caractérise son mandat de Roi ainsi que l'exercice de son pouvoir arbitraire transparaissent dans les termes qu'utilise le narrateur pour en décrire la manifestation dans l'exemple qui va suivre. II s'agit respectivement du verbe interdire et de l'adverbe de manière formellement. Dans l'exemple "le Roi l'interdisait formellement » (Mebou, 2009: 7), ces deux indices sont révélateurs de la volonté d'un homme d'incarner, tout seul, le genre masculin au cœur d'un village où existent néanmoins d'autres hommes plus âgés. Les marqueurs du discours injonctif, interdire et formellement, traduisent, en outre, une sorte de redondance dans les propos. Ils ont pour effet de souligner l'intransigeance d'un Roi attachant du prix à la stricte observance de ses instructions. De fait, l'interdiction articule a priori une forme de nonpermissivité, de refus de négocier voire de rejet systématique d'accueillir l'autre ou de le considérer comme son frère. Le petit garçon se révèle donc être une synecdoque pour le genre masculin, en tant qu'il est susceptible de contrecarrer les plans du monarque s'il venait seulement à naître.

Par ailleurs, lorsque l'interdiction est assortie de la mention formellement, elle traduit un arrière-fond de non-lieu en matière de négoce. Comme stratégie d'anticipation convoquée par le Roi dictateur, le machisme lui sert de mobile pour s'éterniser au trône. La manifestation dudit machisme se perçoit dans ce fragment du narrateur: "seules les fillettes pouvaient naître et vivre sur son territoire " (Mebou, 2009: 7). L'usage du verbe de possibilité pouvaient, pour traduire l'éventualité ou l'incertitude, ainsi que l'emploi de l'adjectif possessif son, dévoilent la capacité de nuisance et la flamme discriminatoire qui régentent l'attitude du Roi. Ces contre-valeurs le poussent à manifester ouvertement de l'aversion à l'endroit d'un genre qui ne saurait, en réalité, se passer de l'existence d'un autre : le genre masculin. 


\subsection{L'aversion de l'homme pour un genre : un art de la méchanceté}

L'inertie, de l'avis d'Olinga, "c'est principalement trois choses: d'abord le cynisme déterminé et l'égoïsme forcené de quelques-uns puissamment organisés » (2009: 11). Le discours du roi s'inscrit dans cette logique de cynisme caractériel empreint d'égoïsme forcené. Ce discours laisse observer un double motif de rejet déployé à l'endroit de l'autre genre, au regard de la posture tranchée qu'adopte le roi aux fins de réifier l'enfant Plus sage que le roi. Le cynisme du monarque transparaît dans la cruauté de ses propos et la barbarie que révèlent en filigrane ses intentions maléfiques. Conscient d'avoir affaire à un enfant en plein processus de croissance biologique, il ne s'offusque pas de le réveiller tôt le matin pour l'inviter à le suivre dans la forêt. Son cynisme, doublé d'ironie, se perçoit à travers les injonctions qu'il imprime aux mots choisis à l'effet de donner forme à son projet de tuer. II dit au jeune enfant: " Plus sage que le Roi, viens avec moi dans la forêt. Lève-toi, je t'attends dehors " (Mebou, 2009: 10). Ce qu'ignore le Roi, c'est que l'enfant bien avisé sait dès le départ leurs rapports respectifs régis par l'hypocrisie et truffés de pièges visant à l'éliminer. Prévenant, l'enfant prend le soin de marquer secrètement son itinéraire afin de s'y repérer le moment venu. Le narrateur omniscient affirme : " comme il faisait très noir et pour ne pas risquer de se perdre en pleine brousse, il entreprit de verser la cendre sur son passage " (Mebou, 2009: 10). La didactique par anticipation se manifeste alors lorsque l'enfant démontre aux adultes qu'une fois en terrain inconnu, ceux-ci gagneraient à se prémunir contre toute forme de danger afin de ne pas se laisser surprendre. Proactif, Plus sage que le Roi « en se couchant ce soir-là, avait emballé dans un petit sac en plastique de la cendre » (Mebou, 2009: 9-10). Sentant poindre le danger, l'enfant anticipe sur les dispositions à prendre afin de conjurer le mauvais sort. Au cynisme du roi qui "souriait secrètement en se disant que Plus sage que le Roi se perdrait dans cette forêt » (Mebou, 2009: 10), s'oppose la force d'anticipation de l'enfant. Le sentiment d'égoïsme qui définit l'inertie et anime le Roi entre alors en ligne de compte dans les rapports qu'entretiennent le monarque avec ses sujets. Ce dernier reste par exemple convaincu qu'en demandant à l'enfant de grimper sur un arbre et de lui montrer ce que " seuls les sages avaient le droit de voir » (Mebou, 2009 :10), celui-ci se perdrait dans la forêt, se faisant alors dévorer par un animal féroce. Ayant par anticipation marqué son itinéraire, l'enfant descend de l'arbre et suit tout simplement les traces de cendre sur le sol qui conduisent jusqu'au village.

Le lecteur de Béatrice Mebou établit à cet égard une constance dans le comportement du Roi face à ses administrés : sa posture de cacique invétéré. Empreinte d'une force destructrice qui pousse à annihiler autour de lui toute intelligence masculine pouvant éclore, la posture royale articule une forme d'aliénation. Celle-ci permet d'attribuer une signification nouvelle à la voix de Frantz Fanon dont Aimé Césaire se fait l'écho en invitant « les peuples à la liberté et l'homme à la dignité » (dans Gambou, 1986 : 107). La didactique des identités de genre que postule l'enfant s'inscrit dans cette mouvance révolutionnaire. Stratégie de désaliénation, celle-ci prône la 
révolte pacifique. Les réactions successives de l'enfant afin d'éviter les pièges du Roi constituent les ressorts de sa pédagogie. Celle-ci emmène le dictateur à réaliser par lui-même l'étroitesse de son raisonnement. Convaincu de détenir le monopole de la sagesse, persuadé que les épreuves auxquelles il soumet l'enfant mèneront ce dernier à sa perte, le Roi ne s'embarrasse pas de les mettre en pratique. D'ailleurs, les mots qu'il utilise le démontrent : " je te ferai passer des épreuves plus dures que tu ne le penses. [...] Dans deux semaines [...], nous allons creuser un puits de vingt-cinq mètres de profondeur » (Mebou, 2009:11). La position du Roi s'assimile à celle du colonisateur dont parle Albert Memmi lorsqu'il affirme : « le colonisateur dénie au colonisé le droit le plus précieux reconnu à la majorité des hommes : la liberté. [...Car] le colonisé n'est pas libre de se choisir colonisé ou non colonisé » (1973: 115). La présence d'esprit du jeune garçon inaugure la rupture avec la vision du monde patriarcale et sexiste qu'adopte le Roi pour instrumentaliser le peuple. II s'agit surtout pour le monarque de travailler dans le sens de maintenir l'enfant dans l'obscurantisme. Pourtant, la didactique des identités de genre affirme l'émergence d'une vision alternative de la vie qui se veut contre-inertielle.

D'après Olinga, l'inertie, dans son second axe, renvoie à «la résignation et l'indifférence du plus grand nombre, occupé à survivre et retarder l'échéance du trépas inéluctable " (2009: 12). A l'instar de Merveille qui, dans Le Journal intime d'une épouse, s'active sur internet et finit par se remarier à un Blanc afin de s'opposer au mariage polygamique que lui imposent se parents, ou encore du Tribun de la plèbe qui, dans Je vois du soleil dans tes yeux, combat son propre père ministre afin de libérer la jeunesse du Koumkana de la mal gouvernance qui obère l'épanouissement du peuple, ainsi Plus sage que le Roi inscrit-il sa didactique dans le sillage de l'action. Celle-ci se définit comme un contre argument à l'inertie. La didactique en question aurait épousé les axes de la philosophie sur l'inertie d'Olinga si l'enfant s'était un tant soit peu résigné. Mais ce dernier choisit d'opposer à l'orgueil du Roi une ingéniosité mémorable doublée d'une rationalité infaillible. Ainsi, dès que le Roi dévoile son plan d'action, l'enfant excite aussitôt ses méninges et mettant en équation une stratégie alternative afin de contourner le piège tendu. Suggestif comme à son habitude, le narrateur révèle la technique adoptée par le gamin comme il suit: « lorsqu'il sortit du palais et que tout le monde se fut éloigné, il commença à creuser dans le sol un sentier qui menait jusqu'à l'endroit fixé par le Roi » (Mebou, 2009: 12). L'enseignement que prodigue l'enfant mystérieux conduit à l'idée qu'au XXlème siècle, il est insensé d'envisager l'épanouissement d'un seul genre, le masculin, dans l'occultation de l'autre genre, le féminin. Le Roi donne de méditer ce postulat dans le récit de Mebou. Fidèle à son cynisme habituel, il ordonne de verser des dizaines de litres d'eau bouillante dans le trou et de l'y faire fermer. II pousse loin son machiavélisme en célébrant la mort de l'enfant. Le narrateur omniscient affirme: " la fête dura toute la nuit et le lendemain " (Mebou, 2009: 12). La didactique des identités de genre dont se veut garant l'enfant postule aussi de rire de la vie au lieu de laisser libre cours à la vengeance comme modalité de réponse au mal subi. Suite à sa résurrection 
miraculeuse, l'ironie caractérisant les propos de Plus sage que le Roi illustre bien ce point de vue. L'enfant nargue le Roi en ces mots sertis d'une tonalité injonctive : «Tenez, mon Roi, je suis encore vivant » (Mebou, 2009: 13).

Toutefois, comme le soutient Barbéris, par-delà sa dimension explicite, le texte comporte et articule un implicite. Pour lui, « un texte n'est pas fait que de chose en clair et qu'on n'avait pas pu ou voulu voir. II est aussi une arcane qui dit le sociohistorique par ce qui ne peut paraître qu'esthétique, spirituel ou moral » (1990: 140).

\section{L'implicite ou l'esthétisation de la problématique du genre}

Dans cette deuxième partie, on démontre que l'écrivain stylise les réalités de la vie car, « par le travail de l'écriture, il [le texte] produit un autre sens " (Mitterand, 1980: 7). Dans son approche de l'implicite, Barbéris suggère que soient décryptées "les situations de blocage et d'impasse ", les "transgressions formelles » et la trigraphie HISTOIRE-Histoirehistoire " (1980: 140-143). Dans la présente étude, nous ne nous intéressons qu'au deuxième aspect portant sur les transgressions formelles.

2.1. Les transgressions formelles

Elles sont de trois ordres dans le cadre de cette étude : le recours au phénomène de l'intergénéricité, l'intermédialité et la prégnance des questions rhétoriques.

L'intergénéricité se trouve au cœur du sens. Elle relève du phénomène de l'hybridité langagière. Elle renvoie au mélange de genres dans un texte littéraire. Dans cet espace d'expression, les genres « s'y [...] font écho et progressent ensemble dans une cohérence certaine que le texte exige " (Aissaoui, 2015: 35). En d'autres termes, l'intergénéricité " étudie les processus de production de sens provoqués par l'union ou l'affrontement de deux genres, par l'entremise de stratégies diverses " (Binette, 2013: 19). Dans le texte scruté, les genres onirique et musical se côtoient harmonieusement.

Le conte écrit se définit comme un récit court en prose. La spécificité de ce genre réside dans un double ancrage diégétique : la narration et la fictionnalisation des événements. S'y trouvent mêlés, des faits réels et des éléments relevant du fantastique. Le conte écrit se distingue ainsi par son caractère merveilleux en ceci que « les contes plongent l'Homme dans un monde imaginaire qui a une logique différente de celle de la vie ordinaire : c'est l'univers où les symboles développent leurs possibles " (Yao konan, 2017: 19-20). Le conte mebousien s'enracine dans le fantastique s'agissant par exemple des conditions atypiques qui entourent la naissance de son personnage phare, l'enfant mystérieux. Le destinateur informe que sa mère " vit en songe qu'elle allait donner naissance à un petit garçon » (Mebou, 2009: 7). Le genre onirique déploie ainsi ses marques dans le conte à travers l'épisode évoquant la mère enceinte. Celle-ci reçoit du futur bébé mystérieux une série de doléances visant à défier l'autorité du Roi phallocrate. Dans un style indirect visant à paraphraser les propos du petit garçon, le narrateur rapporte : « ce petit garçon, bien qu'étant encore dans 
le ventre, pria sa mère de le désigner à sa naissance sous le nom de Plus sage que le Roi. II supplia sa mère de n'avoir aucune crainte à le faire » (2009: 7).

Pour sa part, le genre musical dont le chant est le principal ressort, ponctue les marques de bravoure de l'enfant chaque fois qu'en héros, il se sort d'un piège mortel tendu par le Roi afin de l'éliminer et, avec lui, tout le genre masculin. La particularité du chant qu'entonne à chaque fois l'enfant mystérieux réside dans ce qu'il est presque toujours le même. Le narrateur informe des lieux de déploiement du chant qu'on entend: « vers le château royal »; « devant la porte du Roi » et « vers le palais » $(2009: 11 ; 13 ; 16)$. Le rôle du chant est de tourner le monarque en dérision à l'instant même où, croyant en avoir fini avec l'enfant, il l'entend claironner au loin. Par ailleurs, la mélodie chantée se caractérise par une verve sarcastique marquée du sceau d'une emphase provocatrice introduite par le présentatif c'est. Ladite emphase traduit l'idée de défiance qui anime l'enfant face au tortionnaire sexiste: «Crac ! Crac ! C'est moi Plus sage que le Roi ; Crac ! Crac ! Plus sage que le Roi est de retour !» (2009: 11).

En-deçà de l'intergénéricité, un autre code textuel permet de montrer que «c'est dans la forme même que le romancier [le nouvelliste aussi] donne au mode d'existence sociale de ses personnages, [...] que se glisse le geste idéologique » (Mitterand, 1980: 6). L'inscription textuelle du phénomène de l'intermédialité justifie un tel postulat.

L'intermédialité est un indice de significativité en tant qu'approche textuelle dont l'idée force est qu'une œuvre littéraire a la possibilité de créer des relations intermédiales en exploitant ses propres caractéristiques médiatiques. Se basant sur les travaux de Werner Wolf (1999), on peut dire, avec Rajewsky, que l'attention du lecteur est portée sur une forme d'intermédialité dite intracompositionnelle. Celle-ci suppose une participation indirecte des médias non-littéraires au sein du média dominant qu'est, dans notre cas, le conte écrit. Autant affirmer que le concept d'intermédialité résulte d'une mobilité paradigmatique amenée d'abord par " les nouvelles interactions post-modernes entre les médias et les productions médiatiques " (Müller, 2006: 101), ainsi que par un changement de focalisation de la recherche, qui s'est graduellement tournée vers la matérialité de la communication. Müller résume la naissance de la recherche sur l'intermédialité telle qu'on la conçoit aujourd'hui en expliquant que « le point de départ de cette approche fut la nécessité de tenir compte du fait que concevoir les médias comme des monades isolées était devenu irrecevable » (2006: 100).

Le texte de Mebou est parsemé de gravures ou d'illustrations picturales qui reproduisent quelques scènes du conte relaté. II s'agit pour la nouvelliste de rappeler l'intrusion voulue ou l'interaction féconde entre littérature et peinture. Considérée à la fois comme art et média, la peinture médiatise un mode d'être ou de faire sociétal. Parfois, est schématisé le face-à-face représentant la furie du Roi confrontée à l'hilarité de l'enfant qui s'en moque. Le portrait du Roi exhibe la profondeur de son cynisme. II dépasse, voire surclasse la taille entière de l'enfant qui lui tient tête. II s'agit pour l'auteur de montrer que dans la cité des hommes, les genres sont 
complémentaires si tant est que le monde se bâtit lui-même autour de l'idée de cet impératif de complémentarité des uns vis-à-vis des autres. La couronne du Roi symbolise ce pouvoir dont il sur-abuse, tandis que la tête nue du petit dessine les trajectoires d'une cité neuve construite autour des vertus telles que l'humilité, la convivialité et la charité ou l'altruisme. Telles sont les nouvelles orientations que suggère l'enfant à travers sa didactique différenciée. Pour prendre corps, celle-ci transite par l'assomption d'une posture neuve dont le constructionnisme se veut la clé de voûte.

\subsection{Le conte écrit à l'épreuve du constructionnisme}

L'essentialisme biologique sert de base idéologique au ségrégationnisme parce qu'il s'enracine dans des considérations discriminatoires. Celles-ci mettent en avant l'essence ou la nature des individus, au détriment de leur culture. Ainsi, si le primat de l'essence sur l'existence définit l'idéologie essentialiste, c'est plutôt le sexe social qui caractérise le constructionnisme. Aussi, pour les constructionnistes, n'existe-il ni une essence féminine, ni une essence masculine, mais un sexe biologique qui n'influe pas ou très peu sur la personnalité (Nnomo, 2013: 23). Autant affirmer que le constructionnisme rejoint les perspectives épistémologiques dessinées par l'antinaturalisme féministe dont la visée se résume à un défi : "en finir avec la nature en réduisant totalement le biologique au socio-culturel » (Kraus, 2005: 39). Ce défi est trop grand pour n'être relevé que par les seuls efforts du personnage éponyme Plus sage que le Roi. Le conte de Mebou s'exhibe, dès lors, comme une plateforme didactique où se déploie à la fois le plaidoyer pour un plus grand respect des droits humains, ainsi que l'hymne à la bonne gouvernance et au vivre ensemble.

Le plaidoyer en appelle au respect scrupuleux des droits humains. Dans son ouvrage L'Arrangement des sexes, Claude Zaidman montre que « le masculin et le féminin forment un système (2002: 15). Les deux genres émergent dans une relation de confluence marquée par la complémentarité qui rappelle à Goffman l'urgence d'une " société genrée ". Ainsi, autant on souligne à la suite de De Beauvoir qu'« on ne naît pas femme on le devient » (1949: 13), autant on devrait affirmer, mutatis mutandis, qu'on ne naît pas homme, on le devient. Le constat est donc établi d'affirmer à la suite de Butler que le genre est dans le trouble. C'est ce truisme qui suscite la naissance mystérieuse de l'enfant Plus sage que le Roi dans un pays lointain. Le défi qui définit les traits son existence juvénile procède de sa volonté de déconstruire impérativement un système sexiste qui réifie le genre humain. Dès l'ouverture du conte, le narrateur replonge le lecteur dans les dédales d'un système phallocratique outrageant, qui muselle, réifie et ostracise le citoyen : « dans un pays lointain, les petits garçons n'avaient pas le droit de vivre. Le Roi l'interdisait formellement. Seules les fillettes pouvaient naître et vivre sur son territoire » (Mebou, 2009: 7). Pour le Roi, la femme symbolise la quiétude au plan politique. Elle ne saurait donc aucunement attenter à son pouvoir en tant que sexe faible. Projetant de s'éterniser au pouvoir, le Roi ne voit aucune menace en la femme qu'il l'appréhende, selon le mot de Simone de Beauvoir, comme «l'inessentiel ». 
Voilà pourquoi le monarque sexiste tient à éliminer l'enfant mâle du fait que ce dernier représente une menace pour l'éclosion de la justice sociale et l'épanouissement des identités. Pourtant, la femme s'affirme effectivement comme «l'autre au cœur d'une totalité dont les deux termes sont nécessairement l'un et l'autre " (Beauvoir, 1949: 20). Après une énième épreuve visant à éliminer Plus sage que le Roi, le monarque tue inconsciemment ses propres filles à l'issue d'une ruse mise sur pied par le « terrible garçon » (2009:18). Qui pis est, l'enfant piège le Roi lui-même à l'insu de tous et finit par le tuer par noyade avant de s'adresser au peuple du haut d'un arbre, à travers l'usage d'un certain nombre de questions rhétoriques.

Pour Jean-Jacques Robrieux, l'interrogation rhétorique, encore appelée question de style ou question rhétorique, « n'appelle même pas de réponse, tant la réaction attendue du public est considérée, même de manière forcée, comme évidente " (2000: 116). Ce mode de communiquer est l'apanage de Plus sage que le Roi qui, dans un style ironique dominé par une emphase introduite par le présentatif "c'est », déclare au peuple apeuré: "C'est moi-même ! J'ai tué votre Roi et je suis venu à son deuil. N'était-il pas méchant? [...] Pourquoi ne voulait-il pas voir naître les garçons dans son royaume? Avait-il peur d'être succédé ? N'ai-je pas raison si je vous disais que votre Roi n'a été qu'un assassin pendant son long règne ? » (Mebou, 2009: 18). En précisant que «toute la population présente resta figée » (18), le narrateur confirme la nature de l'interrogation usitée. La fin du règne du Roi dictateur instaure une ère nouvelle : celle de la bonne gouvernance et du vivre ensemble.

En tant qu'hymne à la bonne gouvernance et au vivre ensemble, « le conte [...écrit] est un genre chaleureux qui constitue le limon des relations humaines. Son contenu idéologique vise d'abord et avant tout l'apprentissage de la vie sociale " (Konan, 2017: 29-30). Telles sont les bases de la bonne gouvernance dont les objectifs cadrent avec l'épanouissement optimal des citoyens. Bien gouverner, c'est respecter ses semblables, quel que soit leur genre; c'est aussi observer les règles primaires qui régissent l'éthique, entendue comme "l'ensemble des concessions, des convenances et des conventions qui favorisent le devoirvivre ensemble dans la société » (Mvogo, 2008: 189). En éliminant le Roi du système gouvernemental qui donne libre cours au sacrifice humain, Plus sage que le Roi affranchit le peuple du diktat qui l'enserre. Ce faisant, il contribue à réguler la vie au sein du pays en ouvrant la voie à une amorce du vivre ensemble. La mort du Roi coïncide avec l'avènement d'une ère nouvelle au sein de laquelle "le genre précède le sexe " (Delphy, 2000: 19). II s'agit pour tous les citoyens de prendre une part active à l'avènement du développement du pays en suscitant des hommes neufs mus par la volonté de changer de paradigme gouvernemental. II s'agit d'arguer que «l'heure est venue de rassembler tout le potentiel humain pris dans sa construction sociale et sa force interactive » (Nnomo, 2004: 385). Avant de prendre le pouvoir, toutes choses qui étaient loin de constituer son objectif, l'enfant entreprend une série de concessions qui «font appel au dialogue, à la négociation, à la tolérance, à l'acceptation de l'autre avec ses 
insuffisances et ses qualités » (Mvogo, 2008: 189). C'est à cela qu'invite la bonne gouvernance. Celle-ci se fonde sur des convenances que Mvogo qualifie de «bienséances sociales». Pour lui, elles renvoient « implicitement au savoir-vivre pour soi et avec les autres » (2009:189). Ainsi, une fois fait Roi, l'enfant prend un train de mesures androgynes visant à l'opérationnalisation de ce que Varikas conçoit comme l'« éloge du neutre ». II s'agit d'accorder une égale valeur aux genres afin de mettre l'accent sur les compétences des uns et des autres plutôt que sur leur essence ou leur nature d'homme ou de femme. Voilà pourquoi l'enfant entreprend de semer au sein du pays le bon grain de la paix aux fins de laisser germer le génie de tous au bénéfice de tous. Le narrateur affirme que «le petit garçon devint Roi à l'âge de quatorze ans, et mit fin à l'assassinat des nouveau-nés de sexe masculin » (Mebou, 2009: 18). Cette mesure salutaire porte les germes du vivre ensemble. Sa " conséquence systémique visible dans l'univers fictionnel est un éclatement des murs étroits de la cellule de l'individualisme au profit d'une parfaite coexistence des hommes, des peuples et des races " (Koné et N'golo Aboudou, 2017: 8). Le vivre ensemble ainsi défini épouse l'idée d'une « universalité humaine de condition " (Sartre, 1970: 70). II s'agit d'une nouvelle forme d'humanisme qui intègre la vertu dans le mode de vie des humains. Le vivre ensemble oblige alors le critique à réaffirmer le mode didactique que sécrète le conte, car « la gratuité de l'art ne se conçoit pas. [...] Le conte est, [...] un enseignement [...] qui enseigne aux Hommes les dangers d'un comportement déviant des normes sociales. [...] II s'agit de responsabiliser l'individu, de veiller à l'harmonie en préservant l'équilibre de la société à travers la rectitude des comportements " (Konan, 2017: 30). Le défi que lance le jeune garçon au Roi établit cette vérité distillée dans le conte écrit. L'enfant qui finit par avoir raison d'un Roi en bravant au fil des jours des épreuves atroces montre que seul le vivre ensemble peut conduire un peuple vers les cimes de son développement. D'ailleurs, la conclusion du conte le révèle bien, puisque " durant tout son règne [celui de l'enfant devenu Roi], le royaume vécut heureux » (Mebou, 2009: 18).

\section{Conclusion}

A tout prendre, le conte écrit de Béatrice Laure Mebou illustre la vitalité d'un genre littéraire hybride à mi-chemin entre l'oralité et l'écriture. L'enfant s'y dévoile comme un acteur majeur à travers son implication et son déploiement dans la didactique des identités de genre. En changeant de paradigme, notamment son contexte d'expression, le conte écrit s'émancipe de tout confinement pour finalement s'offrir au monde comme la bouteille à la mer d'Alfred de Vigny. Chaque lecteur est appelé à y tirer le meilleur parti, à y extraire ce que Nicolas Boileau perçoit comme la substantifique moelle. Le conte écrit se positionne ainsi comme un mode de resocialisation fécond de l'humain par l'enfant, par livre interposé. Le commun des mortels hérite surtout des sages enseignements que diffuse à profusion cet outil, notamment les stratégies concourant au vivre ensemble dont ont tant besoin les nations du monde, à l'heure où des mouvements sécessionnistes de tous bords secouent la planète, sacrifiant l'avènement 
de la paix durable et le rêve d'harmonie si chers aux citoyens de la planète, à l'autel de la discrimination sexuelle.

\section{Références bibliographiques}

AISSAOUI, Khadidja. "L'Ecriture de la nouvelle entre intergénéricité, interdiscursivité et interculturel. Le cas d'Enfantement à vif de Raïssi Rachid ", (mémoire de Master, UKM Ouargla, 2015).

BARBERIS, Pierre. "La Sociocritique ». In Introduction aux méthodes critiques pour l'analyse littéraire, edited by Daniel Bergez, Pierre Barbéris, Paris : Bordas, 1990, pp. 121-154.

BEAUVOIR, Simone De. Le Deuxième sexe. Paris, Gallimard, 1949.

BEBEY, Francis. Trois petits cireurs. Yaoundé : CLE, 1989.

BINETTE, Cindy. Intermédialité et intergénéricité dans la télésérie Les Invincibles, Québec : Canada, 2013.

DACY, Elo. L'Actualité de Frantz Fanon. Actes du colloque de Brazzaville. Paris : Karthala, 1986.

KONE, Diakiridia et N'golo Soro Aboudou. De l'altérité à la poétique du vivre ensemble dans la littérature africaine. Paris : L'Harmattan, 2017.

KRAUS, Cynthia (2005), "Avarice épistémique et économie de la croissance : le pas rien du constructionnisme social ». In Le Corps, entre sexe et genre, edited by Hélène Rouch, Elsa Dorlin and Dominique Fougeyrollas Schwebel, Paris : L'Harmattan, 2003, pp. 33-47.

MEAD, Margaret. L'un et l'autre sexe. Paris: folio, 1988.

MEBOU, Béatrice Laure. Plus sage que le roi .... Yaoundé: Ifrikiya, 2014.

Memmi, Albert. Portrait du colonisé précédé de portrait du colonisateur. Paris: Payot, 1973.

MITTERAND, Henri. Le Discours du roman. Paris: PUF, 1980.

MONGO, Pabé, Hane, Khadi and Waberi, Abdourahman Ali. Enfances, Yaoundé : Akoma Mba et Tropiques, 2005.

MÜLLER, Jürgen Ernst. « Vers l'intermédialité. Histoires, positions et options d'un axe de pertinence », Médiamorphoses. L'identité des médias en questions, $\mathrm{n}^{\circ} 16,2006$, pp. 99-110.

MVOGO, Faustin. "Littérature maghrébine: quête, requête ou conquête de l'éthique ? ", In Annales de la FALSH. Identité culturelle et mondialisation, Yaoundé : LGE, 2008, pp. 189-197.

NNOMO, Marcelline. "Le Genre : de la "pensée de la différence" à la postulation d'une catégorie d'analyse », Heuristique, Vol. 1, n² 2, 2013, pp.11-25.

OLINGA, Alain Didier. Propos sur l'inertie. Yaoundé: CLE, 2009.

ORGANISATION INTERNATIONALE DE LA FRANCOPHONIE. Égalité ses sexes et développement. Paris: Jouve, 2002.

PANGOP, Alain Cyr. «Transmission Populaire Au Cameroun : Le Théâtre Et Ses Supports », Notre Librairie. Littérature et développement, n 157, 2005, pp. 68-74.

ROBRIEUX, Jean-Jacques. Rhétorique et Argumentation. Paris: Nathan, 2000.

SARTRE, Jean Paul. L'Existentialisme est un Humanisme. Paris: Nigel, 1970.

WOLF, Werner. Musicalization of Fiction: A Study in the Theory and History of Intermediality, Amsterdam - Atlanta: Rodopi, 1999.

YAO KONAN, Lambert. "Littérature orale africaine et intégration sociale: l'exemple du conte ". In De l'altérité à la poétique du vivre ensemble dans 
Ambigua, Revista de Investigaciones sobre Género y Estudios Culturales, n. ${ }^{\circ}$ 8, 2021, pp. 137-151. ISSN: 2386-8708

la littérature africaine, édited by Kone Diakiridia and N'golo Soro Aboudou. Paris: L'Harmattan, 2017, pp.19-40. 